DISTA-2010

hep-th/yymmnnn

\title{
Pure Spinor Integration from the Collating Formula
}

\author{
P. A. Grassi and L. Sommovigo \\ DISTA, Università del Piemonte Orientale, \\ Via Teresa Michel 11, Alessandria, 15121, Italy \& INFN - Sezione di Torino \\ pietro.grassi@mfn.unipmn.it, luca.sommovigo@mfn.unipmn.it
}

\begin{abstract}
We use the technique developed by Becchi and Imbimbo to construct a welldefined BRST-invariant path integral formulation of pure spinor amplitudes. The space of pure spinors can be viewed from the algebraic geometry point of view as a collection of open sets where the constraints can be solved and a free independent set of variables can be defined. On the intersections of those open sets, the functional measure jumps and one has to add boundary terms to construct a well-defined path integral. The result is the definition of the pure spinor integration measure constructed in term of differential forms on each single patch.
\end{abstract}




\section{Introduction}

One of the main ingredients in the computation of superstring amplitudes is the construction of a path integral measure for the pure spinors [1, 2, 3] (in the text we denote by $\lambda^{\alpha}$ the $10 \mathrm{~d}$ pure spinors satisfying the algebraic equation $\lambda \gamma^{m} \lambda=0$. They are chiral worldsheet bosons and they appear holomorphically in the action and in the path integral).

In the functional integration, we have to distinguish between the integration over the zero modes and the integration over the non-zero modes. The latter can be performed by using the OPE technique and computing the contractions between vertex operators. The former, however, requires much more care because of the pure spinor condition. Actually, the pure spinor space can be seen as an algebraic subvariety of the complex space $\mathbb{C}^{16}$ and, therefore, some techniques of algebraic geometry may be used to construct the amplitudes (see for example [4, 2, 5, 6]). A fundamental observation is the existence of a nowherevanishing holomorphic top-form which has been constructed in term of the pure spinor variables [4]. In terms of such form one can define the integration on the pure spinor space and the physical amplitudes.

However, this integration is subject to some drawbacks: first, the integration on the pure spinor variables is a holomorphic integration (see for example the discussion in [7, 8]) and therefore a holomorphic curve should be given in the pure spinor space (and so far nobody has provided a construction for that path). Nevertheless, in several cases, the algebraic properties of the pure spinors - they carry a representation of the Poincaré group - are sufficient to determine the value of the integration without defining such a curve 1 Second, the space is not compact and it requires a regularization in order to perform meaningful operations.

One way to solve these problems is to introduce new coordinates (in the text we will denote them by $\bar{\lambda}_{\alpha}$ ), leading to the non-minimal formalism [10] where the pure spinor integration is seen as a real integration, as recently discussed in [5, 6]. Indeed, this easily yields the correct coefficients for one- and two-loop amplitudes.

On the contrary, by considering the new variables $\bar{\lambda}_{\alpha}$ as conjugated to the pure spinor variables (satisfying themselves the pure spinor conditions), one can adopt a different point of view: the idea is to construct the pure spinor integration measure from cohomology theory using the isomorphism between the Čech cohomology of the pure spinor space

\footnotetext{
${ }^{1}$ See also 9 . An analogous problem appears in topological strings and holomorphic matrix integrals.
} 
and the de Rham cohomology. For that purpose one needs a partition of unity - which depends upon the conjugated variables-, the measure on a single patch and the Collating Formula [11], which provides the correct machinery to build globally defined forms on a space described in term of its pacthes. Starting from a $p$-form on a given patch and written in terms of local coordinates, one can obtain a corresponding globally defined $p$-form on the entire space. The result is not holomorphic, since the partition of unity is not holomorphic, nevertheless it yields a global top-form to be used to compute the amplitudes. In addition, it may provide a different way to regularize the behavior of the measure at infinity by introducing suitable cut-offs on the patches. This might be very useful to avoid some problems pointed out in literature [2, 8, 12, 13, 14].

The collating formula is a purely mathematical result and its derivation is independent of string theory or quantum field theory. Nonetheless, Becchi and Imbimbo [15] derived it from a path integral approach analyzing the boundary terms needed to define global forms on punctured Riemann surfaces. The path integral approach shows how the jumps in the measure passing from one patch to another yield boundary terms which can be removed by adding contributions to the measure reproducing the collating formula.

In the present paper, we do not discuss the applications of the present formalism and they will be postponed in a subsequent publication dedicated to the amplitude computations with the present formula.

In sec. 2 we present a construction of the measure for algebraic varieties by generalizing the Griffiths' method in the case of non-complete intersections. In addition, we derive the measure in a different way resulting in a vector-valued holomorphic form and we explain the relation with the holomorphic top form. At the end of the section, we construct a real globally defined form. In sec. 3 we discuss the mathematical Collating Formula. In sec. 4 we translate it in term of a BRST formulation and a path integral technique leading to a globally defined real form. In sec. 5 we provide some examples of the application of the collating formula.

\section{Integration on Constrained Spaces}

We consider a set of variables $Z_{i}$ constrained by the algebraic equations $W^{a}(Z)=0$. They describe the $k$-dimensional hypersurfaces denoted by $\mathcal{P}$. The index $a=1, \ldots, q$ runs over the number of polynomials $W^{a}(Z)$ in the variables $Z_{i}$ and $i$ runs over the dimension of the ambient manifold which is assumed to be $\mathbb{C}^{N}$. 
If the space is a complete intersection, the constraints $W^{a}(Z)$ are linearly independent and the differential form

$$
\Theta^{(N-k)}=\epsilon_{a_{1} \ldots a_{N-k}} d W^{a_{1}} \wedge \cdots \wedge d W^{a_{N-k}}
$$

is not vanishing. In this case, $q=N-k$ and the dimension of the surface is easily determined. For example, if the hypersurface is described by a single algebraic equation $W(Z)=0$, the form (2.1) is given by $\Theta^{(1)}=d W$.

On the other hand, if the hypersurface is not a complete intersection, then there exists a differential form

$$
\Theta^{(N-k)}=\mathcal{T}_{A,\left[a_{1} \ldots a_{q}\right]} d W^{a_{1}} \wedge \cdots \wedge d W^{a_{q}} \wedge \eta^{A,(N-k-q)},
$$

where $\eta^{A,(N-k-q)}$ is a set of $N-k-q$ forms defined such that $\Theta^{(N-k)}$ is non-vanishing on the constraints $W^{a}(z)=0$ and $\mathcal{T}_{A,\left[a_{1} \ldots a_{q}\right]}$ is a numerical tensor which is antisymmetric in the indices $a_{1} \ldots a_{q}$. The construction of $\eta^{A,(N-k-q)}$ depends upon the precise form of the algebraic variety. In some cases a general form can be given, but in general it is not easy to find it and we did not find a general procedure for that computation? 2

To construct a global form on the space $\mathcal{P}$ one can use a modification of the Griffiths' residue method [16] by observing that given the global holomorphic form on the ambient space $\Omega^{(N)}=\epsilon^{i_{1} \ldots i_{N}} d Z_{i_{1}} \wedge \cdots \wedge d Z_{i_{N}}$, we can decompose the $\left\{Z_{i}\right\}$ 's into a set of coordinates $Y^{a}=W^{a}(Z)$ and the rest. By using the contraction with respect to $q$ vectors $\left\{\bar{Z}_{i}^{a}\right\}$, the top form for $\mathcal{P}$ can be written as

$$
\Omega^{(k)}=\frac{\iota_{\bar{Z}^{a_{1}}} \ldots \iota_{\bar{Z}^{a_{q}}} \Omega^{(N)}}{\iota_{\bar{Z}^{a_{1}} \cdots \iota^{a^{a_{q}}} \Theta^{(N-k)}}}
$$

which is independent from $\left\{\bar{Z}_{i}^{a}\right\}$ as can be easily proved by using the constraints $W^{a}(Z)=$ 0 . Notice that this form is nowhere-vanishing and non singular only in the case of CY space. The vectors $\left\{\bar{Z}_{i}^{a}\right\}$ play the role of gauge fixing parameters needed to choose a polarization of the space $\mathcal{P}$ into the ambient space.

In the case of pure spinor we have: the ambient form $\Omega^{(16)}=\epsilon_{\alpha_{1} \ldots \alpha_{16}} d \lambda^{\alpha_{1}} \wedge \ldots \cdots \wedge d \lambda^{\alpha_{1} 6}$ and $\Theta^{(5)}=\lambda \gamma^{m} d \lambda \lambda \gamma^{m} d \lambda \lambda \gamma^{m} d \lambda d \lambda \gamma_{m n p} d \lambda$. From these data, we can get the holomorphic top form $\Omega^{(11)}$ by introducing 5 independent parameters $\bar{\lambda}$ and by using the formula (2.3).

\footnotetext{
${ }^{2}$ In the case of Normal Rational Curves (which are not complete intersections), the form is given by $\Theta^{(N-2)}=\epsilon^{I_{1} \ldots I_{q} I_{q+1} \ldots I_{N}} d W_{I_{1}} \wedge \cdots \wedge d W_{I_{q}} \wedge \omega_{I_{q+1}}^{1} \wedge \cdots \wedge \omega_{I_{N}}^{N-q}$ where the vectors $\omega^{r}$ are defined by means of the relations (syzygy) between the constraints $W^{I}$ such that $\omega_{I}^{r}(Z) W^{I}(Z)=0$ for all $r=1, \ldots, N-q$. In this case the vectors $\omega_{I}^{r}(Z)$ are global functions on the space.
} 
The latter is independent from the choice of the parameters $\bar{\lambda}$ (however, some care has to be devoted to the choice of the contour of integration and of the integrand: in the minimal formalism, the presence of delta functions $\delta(\lambda)$ might introduce some singularities which prevent from proving the independence from $\bar{\lambda}$, as was pointed out in [7, 17]).

Using $\Omega^{(k)} \wedge \overline{\Omega^{(k)}}$, one can compute the correlation functions by integrating globally defined functions. When the space is Calabi-Yau, it also exists a globally-defined nowherevanishing holomorphic form $\Omega_{h o l}^{(k \mid 0)}$ such that $\Omega_{h o l}^{(k \mid 0)} \wedge \overline{\Omega_{h o l}^{(0 \mid k)}}$ is proportional to $\Omega^{(k)} \wedge \overline{\Omega^{(k)}}$. The ratio of the two top forms is a globally defined function on the CY space.

In the case of the holomorphic measure $\Omega_{h o l}^{(k \mid 0)}$ the integration of holomorphic functions is related to the definition of a contour $\gamma \in \mathcal{P}$ in the complex space

$$
\left\langle\prod_{A} \mathcal{O}\left(p_{A}\right)\right\rangle=\int_{\gamma \in \mathcal{P}} \Omega^{(k, 0)}\left(Z_{i}\right) \prod_{A} \mathcal{O}_{0}\left(Z_{i}, p_{A}\right)
$$

where $\mathcal{O}\left(Z_{i}, p_{A}\right)$ are the vertex operators of the theory localized at the points $p_{A}$ of the Riemann surface and $\mathcal{O}_{0}\left(Z_{i}, p_{A}\right)$ is the zero-mode component of the vertex operators. They are functions of the coordinates $Z_{i}$ which are reduced to their zero modes (the non-zero mode part is computed by the usual OPE technique).

For instance, let us consider the hypersurface $\sum_{i} Z_{i}^{2}=0$ in $\mathbb{C}^{N}$. This equation can be put in the form $P\left(u_{i^{\prime}}\right)=w z$ where $i^{\prime}$ runs over $i^{\prime}=1, \ldots, N-2$ coordinates and $w, z$ are two combinations of the $Z$ 's. $P\left(u_{i^{\prime}}\right)$ is a polynomial of the coordinates $u_{i^{\prime}}$. For a given $\mathrm{N}$ they are local CY spaces and there exists a globally-defined, nowhere-vanishing holomorphic top form given by

$$
\Omega^{(N-1 \mid 0)}=\frac{d z \wedge d u_{i^{1}} \wedge \cdots \wedge d u_{i^{N-2}}}{z}
$$

In this case, the ambient form is $\Omega^{(N)}=\epsilon_{i_{1} \ldots i_{N}} d Z^{i_{1}} \wedge \cdots \wedge d Z^{i_{N}}$ while the $\Theta$ form is $\Theta^{(1)}=\sum_{i=1}^{N} Z^{i} d Z^{i}$. Again, by introducing the parameters $\bar{Z}$ (and assuming that they transform under a vector representation of $S O(N)$ ), this form can also be written in an $S O(N)$ invariant way by using (2.3)

$$
\Omega^{(N-1,0)}=\frac{\bar{Z}_{i_{1}} \epsilon^{i_{1} \ldots i_{N}} d Z_{i_{2}} \wedge \cdots \wedge d Z_{i_{N}}}{\bar{Z}^{i} Z_{i}}
$$

Note from (2.5) that the measure has a possible pole for $z=0$, however the form of (2.5) depends upon the choice of $\bar{Z}$ and this corresponds to the choice of a patch where the corresponding coordinate does not vanish. Since, in general (as discussed after (2.3)) the measure is independent from the $\bar{Z}$, also the measure given in (2.5) is free from singularities. 
There is another way to construct a measure for these examples which is independent of the choice of the $\bar{Z}$, namely a measure with values in the tangent vector bundle. We define the tensor $T^{(1, N-1 \mid 0,0)}$ which is a holomorphic vector with values in the $N$ - 1 -form space as follows

$$
T^{(1, N-1 \mid 0,0)}=\epsilon^{i_{1} \ldots i_{N}} d Z_{i_{1}} \wedge \cdots \wedge d Z_{i_{N-1}}\left(g^{-1}\right)_{i_{N} k} \partial^{k}
$$

which is compatible with a single constraint $W\left(Z_{i}\right)=0$ and

$$
g^{l k}=\frac{\partial^{2} W}{\partial Z_{l} \partial Z_{k}} .
$$

The relation between (2.6) and (2.7) is given by the equation

$$
\Omega^{(N-1 \mid 0)}=T^{(1, N-1 \mid 0,0)}\left[\ln \left(\bar{Z}^{i} g_{i j} Z^{j}\right)\right],
$$

where the differential operator acts on the globally defined function $\ln \left(\bar{Z}^{i} g_{i j} Z^{j}\right)$, which is singular only for $\bar{Z}^{i} g_{i j} Z^{j}=0$. In the present case there is a single constraint and therefore a single set of parameters $\bar{Z}^{i}$ and we identify them with the complex conjugated to $Z^{i}$.

In the case of pure spinors the measure is given by

$$
T^{(3,11 \mid 0,0)}=\epsilon_{\alpha_{1} \ldots \alpha_{16}} d \lambda^{\alpha_{1}} \wedge \cdots \wedge d \lambda^{\alpha_{11}}\left(\gamma^{m n p}\right)^{\alpha_{12} \alpha_{1} 3}\left(\gamma_{m} \partial_{\lambda}\right)^{\alpha_{14}}\left(\gamma_{n} \partial_{\lambda}\right)^{\alpha_{16}}\left(\gamma_{p} \partial_{\lambda}\right)^{\alpha_{16}}
$$

which is covariant under Lorentz transformations and, acting on the function $\ln \left(\bar{\lambda}_{\alpha} \lambda^{\alpha}\right)$, it is related to the expression obtained in the non-minimal formalism [10].

Until now, we have constructed global holomorphic measures for the integration on the zero modes. However, as we have already pointed out, the integration over such a measure requires a holomorphic curve. Nevertheless we can avoid such a trouble by constructing a real measure (which amounts to a specific choice of integration countour). We observe that, if we define the measure on the conjugated variables using the rule (2.5)

$$
\bar{\Omega}^{(0,0 \mid 0, N-1)}=\frac{Z_{i_{1}} \epsilon^{i_{1} \ldots i_{N}} d \bar{Z}_{i_{2}} \wedge \cdots \wedge d \bar{Z}_{i_{N}}}{\bar{Z} \cdot Z}
$$

where we used the variables $Z^{i}$ as gauge parameters in (2.5), we can act on it with the differential operator $T^{(1, N-1 \mid 0,0)}$ to get

$$
T^{(1, N-1 \mid 0,0)} \wedge\left[\bar{\Omega}^{(0,0 \mid 0, N-1)}\right]=d Z_{i_{2}} \wedge \cdots \wedge d Z_{i_{N}} \epsilon^{i_{1} \ldots i_{N}} G_{i_{1} j_{1}} \epsilon^{j_{1} \ldots j_{N}} d \bar{Z}_{j_{2}} \wedge \cdots \wedge d \bar{Z}_{i_{N}}
$$

where

$$
G_{i_{1} j_{1}}=\left(\frac{\delta_{i_{1} j_{1}} \bar{Z} \cdot Z-\bar{Z}_{i_{1}} Z_{j_{1}}}{(\bar{Z} \cdot Z)^{2}}\right)
$$


is the Fubini-Study metric on $\mathbb{P}^{N-1}$ written in terms of the homogenous coordinates. A similar result has been obtained by Gomez in [5]. This measure has the advantage of being real and globally defined, but it is clearly not holomorphic and it does not require a path to be specified.

This construction of the measures does not make use of the decomposition of the manifold in patches but, if we are willing to abandon the holomorphicity, we can build the top forms from a completely different point of view. Namely, we start from the measure on a single patch, which is the usual flat measure written in terms of a set of convenient coordinates, and we construct the global measure using the Collating Formula by gluing the contributions coming from the different patches.

\section{Collating formula}

A fundamental theorem in cohomological theory is [11]

Theorem: If $\mathfrak{U}$ is a good cover of the manifold $\mathcal{M}$, then the de Rham cohomology $H_{D R}^{*}(\mathcal{M})$ of $\mathcal{M}$ is isomorphic to the Čech cohomology $H^{*}(\mathfrak{U}, \mathbb{R})$ of the good cover

$$
H_{D R}^{*}(\mathcal{M}) \simeq H^{*}(\mathfrak{U}, \mathbb{R})
$$

where the inclusions

$$
\mathcal{M} \leftarrow \mathcal{U}_{I} \leftleftarrows \mathcal{U}_{I J} \leftleftarrows \mathcal{U}_{I J K} \cdots
$$

include $\mathcal{U}_{I}$, the open sets of the good cover into the multiple intersections of the open sets $\mathcal{U}_{I_{1} \ldots I_{n}} \equiv \underset{i=1 \ldots n}{\cap} \mathcal{U}_{I_{i}}$.

The meaning of the present theorem is the following: on one hand, the differential geometry of forms establishes an exact sequence in the complex $0 \rightarrow \Omega^{*}(\mathcal{M}) \stackrel{r}{\rightarrow} C^{*}\left(\mathfrak{U}, \Omega^{*}\right)$, where $r$ is the restriction map which restricts the exterior algebra $\Omega^{*}(\mathcal{M})$ to the complex of cochains of differential forms $C^{*}\left(\mathfrak{U}, \Omega^{*}\right)=\oplus_{p, q \geq 0} C^{p}\left(\mathfrak{U}, \Omega^{q}\right)$ on the cover of $\mathcal{M}$. On the other hand, starting from the combinatorics of the cover $\mathfrak{U}$, one can compute the Mayer-Vietoris sequence of the complex $0 \rightarrow C^{*}(\mathfrak{U}, \mathbb{R}) \rightarrow C^{*}\left(\mathfrak{U}, \Omega^{*}\right)$. Finally, in the double complex the two cohomologies are mixed. In this way, one proves that $H_{D R}^{*}(\mathcal{M}) \simeq$ $H_{D}\left(C^{*}\left(\mathfrak{U}, \Omega^{*}\right)\right)$ using the fact that the de Rham cohomology of $\mathcal{M}$ is isomorphic to the cohomology of the double complex. In addition, if $\mathfrak{U}$ is a good cover, the Cech cohomology is also isomorphic to the cohomology of the double complex $H^{*}(\mathcal{M}, \mathbb{R}) \simeq H_{D}\left(C^{*}\left(\mathfrak{U}, \Omega^{*}\right)\right)$, and therefore it follows the isomorphism between the de Rham and the Čech cohomology. 
The main point is that this isomorphism provides a way to compute the de Rham cohomology by means of combinatorics of a good cover (we refer to [11] for a complete discussion regarding the existence of good cover and its implications). Here we only describe the crucial formula providing the explicit isomorphisms between the two cohomologies. Given $f$ a chain map

$$
f: C^{*}\left(\mathfrak{U}, \Omega^{*}\right) \rightarrow \Omega^{*}(\mathcal{M})
$$

such that $f \circ r=1$ and $r \circ f$ is chain homotopic to the identity. The map $f$ provides the main ingredient for collating together a Čech-de Rham cochain into a global form. This is given by the Collating Formula. For the description of the collating formula we need to define a homotopy operator $K$ by introducing the partition of unity $\rho_{I}$ (such that every point of $\mathcal{M}$ has a neighborhood in which $\sum_{I} \rho_{I}$ is a finite sum, $\sum_{I} \rho_{I}=1$ and defined such that $\left.\operatorname{supp}\left[\mathcal{U}_{I}\right] \subset \mathcal{U}_{I}\right)$ and $K$ acting on a $p$-cochain $\omega_{I_{0} \ldots I_{p}}$ gives

$$
(K \omega)_{I_{0} \ldots I_{p-1}}=\sum_{I} \rho_{I} \omega_{I I_{0} \ldots I_{p-1}}
$$

If $\delta$ is the Čech operator $(\delta \omega)_{I_{0} \ldots I_{p}}=\sum_{i=1}^{p}(-)^{i} \omega_{I_{0} \ldots \hat{I}_{i} \ldots I_{p}}$ (where hatted index must be omitted), then the homotopy operator $K$ satisfies the relation $K \delta+\delta K=1$. The last ingredients are the differential operators $D^{\prime \prime}=(-1)^{p} d$, acting on the complex $C^{*}\left(\mathfrak{U}, \Omega^{*}\right)$ where $p$ is the degree of the cochain, and $D=\delta+D^{\prime \prime}$.

With these ingredients we can provide the Collating Formula. Let $K$ be the homotopy operator defined above and $\alpha=\sum_{i=0}^{n} \alpha_{i}$ is an $n$-cochain such that $D \alpha=\beta=\sum_{i=0}^{n+1} \beta_{i}$. They satisfy the following descent equations

$$
d \alpha_{0}^{p}=\beta_{0}^{p+1}, \quad d \alpha_{1}^{p-1}+\delta \alpha_{0}^{p}=\beta_{1}^{p}, \quad \ldots \quad d \alpha_{p}^{0}+\delta \alpha_{p-1}^{1}=\beta_{p}^{1}, \quad \delta \alpha_{p}^{0}=\beta_{p+1}^{0},
$$

where the differential operator $d$ increases the form number and the difference operator $\delta$ increases the co-chain number. If we act with the homotopy operator $K$ on the last element of the descent equation, we get $K \delta \alpha_{p}^{0}=K \beta_{p+1}^{0}$, and using the algebraic relation between $\mathrm{K}$ and $\delta$, it yields $\alpha_{p}^{0}=\delta K \alpha_{p}^{0}+K \beta_{p+1}^{0}$. In the same way, inserting this result in the next-to-the-last equation in (3.5), we obtain the next cochain $\alpha_{p-1}^{1}$ in terms of $\beta$ 's and $\alpha_{p}^{0}$. Proceeding in this way we get the following final formula

$$
f(\alpha)=\sum_{i=0}^{n}\left(-D^{\prime \prime} K\right)^{i} \alpha_{i}-\sum_{i=1}^{n+1} K\left(-D^{\prime \prime} K\right)^{i-1} \beta_{i} \in C^{0}\left(\mathfrak{U}, \Omega^{n}\right) .
$$

This is a global form satisfying the above mentioned properties. We refer to [1] for the proofs and the discussion. In the present form, this formula is not very useful for 
our purposes since we do not know the various terms and they must be computed from (3.6). For that purpose it is convenient a path-integral derivation adapted to the problems discussed in the previous section. In that way the all ingredients in the above formula are specified and the global form can be easily derived 3

The main idea is that the path integral has a jump passing from one patch to another and this jump is seen as an anomaly in the Ward Identity. The contributions needed to remove such anomaly terms are indeed the addends in (3.6).

\section{4 (Path Integral) Collating Formula}

We translate the above algebraic-differential derivation into a path integral formulation adapted to sigma models and string models.

Our model is defined by the action $S[Z]=\int_{\Sigma} d^{2} z \mathcal{L}(Z(z, \bar{z}))$ where $\mathcal{L}(Z(z, \bar{z}))$ is the Lagrangian of the sigma model and $Z(z, \bar{z}): \Sigma \rightarrow \mathcal{M}$ maps the worldsheet $\Sigma$ into the target space $\mathcal{M}$. We assume that the target space is a complex manifold with a Kähler form $K=K_{i \bar{j}} d Z^{i} \wedge d \bar{Z}^{\bar{j}}$, where $\bar{Z}^{\bar{i}}$ are the complex conjugated variables. The action needs not be real and we assume to be starting with a holomorphic Lagrangian $\mathcal{L}(Z)$; the gauge-fixing will possibly involve the complex conjugated coordinates.

In general, the target space $\mathcal{M}$ can be better described in terms of an atlas, namely in terms of a given set of open sets $\mathcal{U}_{I}$ and a system of coordinates $Z_{(I)}$ 's on each of them. The form of the target space is parameterized by the transition functions between the different patches

$$
h_{J I}:\left(\mathcal{U}_{I}, Z_{(I)}\right) \longrightarrow\left(\mathcal{U}_{J}, Z_{(J)}\right)
$$

such that $h_{J I}\left(\mathcal{U}_{I}\right) \subset \mathcal{U}_{J}$ and $h_{J I}\left(Z_{(I)}\right)=Z_{(J)}$. We assume that the transition functions $h_{I J}$ are holomorphic functions of $Z$ 's. (A partition of unity for the atlas $\bigcup_{I}\left(\mathcal{U}_{I}, Z_{(I)}\right)$ can be given by $\rho_{I}=\frac{\bar{Z}_{I} Z_{I}}{Z \cdot Z}$, with $\bar{Z} \cdot Z=\bar{Z}^{i} Z_{i}$, and where we have assumed that on the patch $I$ the coodinate $Z_{i}$ with $i=I$ is different from zero).

The local observables of the theory are given by the vertex operators $\mathcal{O}_{A}(Z)$ (where $A$ runs over the whole set of vertex operators) and they must be globally defined on $\mathcal{M}$, holomorphic and BRST invariant.

\footnotetext{
${ }^{3} \mathrm{In}[15]$ the solution of the descent equation is obtained by introducing a set of anticommuting auxiliary variables $\xi^{\alpha}$ and rewriting the Čech operator as $\delta=\sum_{\alpha} \xi^{\alpha}$ and the homotopy operator as $K=\sum_{\alpha} \rho_{\alpha} \partial_{\xi^{\alpha}}$.
} 
The correlation functions are computed as follows

$$
\begin{gathered}
\left\langle\prod_{A} \mathcal{O}_{A}(Z)\right\rangle=\left.\prod_{A} \frac{\delta}{\delta \eta_{A}} Z\left[\eta_{A}\right]\right|_{\eta=0} \\
Z\left[\eta_{A}\right]=\int \mathcal{D} Z e^{-S[Z]+\sum_{A} \eta_{A} \mathcal{O}_{A}(Z)}
\end{gathered}
$$

$\eta_{A}$ are the sources for the observables and $\mathcal{D} Z$ is the naive functional integration measure. This formula is intrinsically badly-defined if there is not a global coordinate system, since the naive integration measure $\mathcal{D} Z$ makes sense only in the open patches $\mathcal{U}_{\mathcal{I}}$. Moving from one patch to another, the integration measure could receive contributions from the intersections of the different patches and these contributions are fundamental to provide a functional measure which is globally defined on the whole target space.

Therefore, we start from a different expression

$$
Z\left[\eta_{A}\right]=\sum_{I} \int \mathcal{D} Z_{(I)} e^{-S\left[Z_{(I)}\right]+\sum_{A} \eta_{A} \mathcal{O}_{A}\left(Z_{(I)}\right)}
$$

where the integration is done patch by patch and we use the naive integration on any single patch $\mathcal{D} Z_{(I)}$. If we decompose the field $Z_{(I)}$ in terms of zero modes and non-zero modes, the naive functional integration measure is given by

$$
\mathcal{D} Z_{(I)}=\left|d Z_{(I) 0}^{1} \wedge \cdots \wedge d Z_{(I) 0}^{n} \prod_{k=1}^{\infty} d Z_{(I) k}^{1} \wedge \cdots \wedge d Z_{(I) k}^{n}\right|^{2} .
$$

The observables $\mathcal{O}_{A}\left(Z_{(I)}\right)$ are defined by means of the restriction map (see sec. 3) from the globally defined observables $\mathcal{O}_{A}(Z)$ and the action $S\left[Z_{(I)}\right]$ is defined by the restriction map from the action $S[Z]$.

Now, we assume that the non-zero modes are treated with the conventional OPE technique, so that we can consider only the zero modes of the theory. We write the integrand as an $(n \mid n)$ form

$$
\omega_{I}^{(n \mid n)}=\bigwedge_{i=1}^{n}\left|d Z_{(I) 0}^{i}\right|^{2} F\left(Z_{(I) 0}, \eta_{A}\right)
$$

where $F\left(Z_{(I) 0}, \eta_{A}\right)$ is the result of the non-zero modes integration. This formula shows that there are two sources of patch dependence: one in the integration measure and the other in the integral of the non-zero modes (since the decomposition between zero modes and non-zero mode depends upon the choice of the patch). Therefore, it is convenient to 
introduce an auxiliary variable $x^{i}$ and to rewrite the above expression as

$$
\begin{aligned}
\omega_{(I)}^{(n \mid n)} & =\int \bigwedge_{i=1}^{n}\left|d x^{i} \delta\left(x^{i}-Z_{(I) 0}^{i}\right)\right|^{2} \bigwedge_{i=1}^{n}\left|d Z_{(I) 0}^{i}\right|^{2} F\left(Z_{(I) 0}, \eta_{A}\right) \\
& =\int \bigwedge_{i=1}^{n}\left|d x^{i}\right|^{2} F\left(x, \eta_{A}\right) \bigwedge_{i=1}^{n}\left|d Z_{(I) 0}^{i} \delta\left(x^{i}-Z_{(I) 0}^{i}\right)\right|^{2}
\end{aligned}
$$

The Dirac delta function localizes the expression $F\left(x, \eta_{A}\right)$ on a single patch but, now, the patch dependence is entirely in the last factor which can be written as

$$
\begin{aligned}
\omega_{(I)}^{(n \mid n)} & =\int \bigwedge_{i=1}^{n}\left|d x^{i}\right|^{2} F\left(x, \eta_{A}\right) \int\left|d B_{i}\right|^{2}\left|d \bar{C}_{i}\right|^{2} e^{B_{i}\left(x^{i}-Z_{I}^{i}\right)+\bar{C}_{i} d Z^{i}+\text { c.c. }} \\
& \equiv \int \bigwedge_{i=1}^{n}\left|d x^{i}\right|^{2} F\left(x, \eta_{A}\right) \int\left|d B_{i}\right|^{2}\left|d \bar{C}_{i}\right|^{2} \omega_{(I)} \\
& \omega_{(I)}=e^{d\left[\bar{C}_{i}\left(x^{i}-Z_{I}^{i}\right)\right]+\text { c.c. }}
\end{aligned}
$$

where we have introduced $n$ pairs of auxiliary fields $B_{i}$ and $\bar{C}_{i}$ with the property that $d B_{i}=0, d \bar{C}_{i}=B_{i}$ and $d Z_{I}^{i}$ is the usual basis of 1 -forms ( $d$ is automatically nilpotent). The function $\bar{C}_{i}\left(x^{i}-Z_{I}^{i}\right)$ plays the role of a gauge-fixing fermion with negative ghost number (carried by the $\bar{C}_{i}$ ) and it selects the patch on which the functional is evaluated. Notice that at this point one can substitute the Dirac delta function with a smooth expression. Now, our next problem is how to construct a globally defined expression for $\omega_{(I)}$.

Following [19] we study the variation of the $\omega_{I}$ 's changing the patch and we get

$$
\begin{aligned}
\omega_{(I)}-\omega_{(J)} & =e^{d\left(\bar{C}_{i}\left(x^{i}-Z_{I}^{i}\right)+\text { c.c. }\right)}-e^{d\left(\bar{C}_{i}\left(x^{i}-Z_{J}^{i}\right)+\text { c.c. }\right)}= \\
& =d\left[\bar{C}_{i}\left(Z_{J}^{i}-Z_{I}^{i}\right) \int_{0}^{1} e^{d\left[t \bar{C}_{i}\left(x^{i}-Z_{I}^{i}\right)+(1-t)\left(\bar{C}_{i}\left(x^{i}-Z_{J}^{i}\right)+\text { c.c. }\right]\right.}\right]=d \omega_{(I J)}
\end{aligned}
$$

this can be easily proven by observing that $d$ acts only on the factor in front of the integral, since the argument is $d$-closed, and the actual effect of the differential operator on the prefactor is to give a total derivative w.r.t. $t$. Hence, the difference between $\omega_{(I)}$ and $\omega_{(J)}$ is $d$-exact and this allows us to employ the descent equations technique to derive a ladder of forms $\omega_{\left(I_{1} \ldots I_{p}\right)}$ defined on the $p$-intersections $\mathcal{U}_{\left(I_{1} \ldots I_{p}\right)}$ of the open sets $\mathcal{U}_{\mathcal{I}}$.

The main point is that, in [18, 19], a prescription is given on how to compute the complete set of forms satisfying the descent equations. The forms $\omega_{\left(I_{1} \ldots I_{p}\right)}$ can be straightforwardly 
written as the product of a prefactor $A$ times an interpolating action $S\left[t_{1}, \ldots, t_{p}\right]$

$$
\begin{aligned}
\omega_{\left(I_{1} \ldots I_{p}\right)} & =A_{I_{1} \ldots I_{p}} \times S\left[t_{1}, \ldots, t_{p}\right] \\
A_{I_{1} \ldots I_{p}} & =\frac{1}{p !} \sum_{\alpha=1}^{p}(-1)^{\alpha+1} \bar{C}_{i_{1}}\left(x^{i_{1}}-Z_{\left(I_{1}\right) 0}^{i_{1}}\right) \ldots \overbrace{\bar{C}_{i_{\alpha}}\left(x^{i_{\alpha}}-Z_{\left(I_{\alpha}\right) 0}^{i_{\alpha}}\right)} \ldots \bar{C}_{i_{p}}\left(x^{i_{p}}-Z_{\left(I_{p}\right) 0}^{i_{p}}\right)+\text { c.c. } \\
S\left[t_{1}, \ldots, t_{p}\right] & =\int_{0}^{1}\left(\prod_{\alpha=1}^{p} d t_{\alpha}\right) \exp \left\{d\left[\sum_{\beta=1}^{p} t_{\beta} \bar{C}_{i}\left(x^{i}-Z_{I_{\beta}}^{i}\right)+\text { c.c. }\right]\right\} \delta\left(\sum_{\alpha=1}^{p} t_{\alpha}-1\right)
\end{aligned}
$$

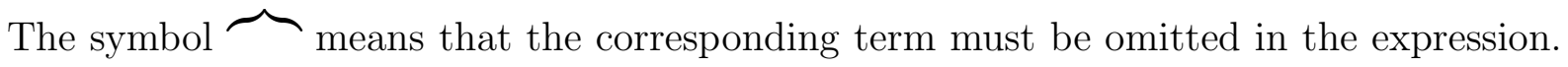
It is easy to verify that they satisfy the descent equations

$$
d \omega_{(I)}^{n}=0, \quad \ldots \quad\left(\delta \omega^{n-p+1}\right)_{\left(I_{1} \ldots I_{p}\right)}=d \omega_{\left(I_{1} \ldots I_{p}\right)}^{n-p}, \quad \ldots \quad\left(\delta \omega_{\left(I_{1} \ldots I_{n}\right)}^{0}\right)=0 .
$$

At order $p$ the prefactor contains $p$ powers of the anticommuting variable $\bar{C}$ so that, in order for the $\bar{C}$ integral not to vanish, $n-p-1$ further powers of $\bar{C}$, which are extracted from the exponential, are needed. The resulting expression is a $n-p-1$ form with ghost number $p$. Notice that assembling the monomials $\left(x^{i}-Z_{I}^{i}\right)$ into a matrix with $n$ rows (the number of independent coordinates on the target space) and $q$ columns (the number of patches of the atlas) the prefactor computes the determinant of its minors. It may happen that from a given order all the determinants vanish. This formula provides a suitable choice for the component of the ladder in the descent equations (each component is defined up to exact terms).

Using the collating formula for $\omega_{(I)}$ we can finally write the globally defined expression

$$
\omega_{\text {global }}=\omega_{(I)}+\sum_{l=1}^{n}\left(-D^{\prime \prime} K\right)^{l} \omega_{(l)}
$$

where we have compactly denoted $\left(-D^{\prime \prime} K\right)^{l} \omega_{(l)}=\left(-D^{\prime \prime} K\right)^{I_{1}} \ldots\left(-D^{\prime \prime} K\right)^{I_{l}} \omega_{\left(I I_{1} \ldots I_{l}\right)}$ and each differential operator $D^{\prime \prime}$ acts on the entire expression on its right.

At this point one can integrate over the anticommuting fields $\bar{C}$ and over the commuting pairs $B_{i}$ and $x^{i}$ leading to an expression which is globally defined providing the correct integrand of (4.2). Notice that, in this way, one selects the form degree needed in the integration. By following again [15], inserting the global form $\omega_{\text {global }}$ in the integration, 
we have

$$
\begin{aligned}
& \int \bigwedge_{i=1}^{n}\left|d x^{i}\right|^{2} F\left(x, \eta_{A}\right) \int\left|d B_{i}\right|^{2}\left|d \bar{C}_{i}\right|^{2} \omega_{\text {global }} \\
= & \int \bigwedge_{i=1}^{n}\left|d x^{i}\right|^{2} F\left(x, \eta_{A}\right) \sum_{q=0}^{n}(-1)^{q} \sum_{I_{0}<I_{1}<\cdots<I_{q}} \int_{\mathcal{C}_{I_{0} \ldots I_{q}}} \omega_{\left(I_{0} \ldots I_{q}\right)}^{n-q}\left(x, Z_{0}\right)
\end{aligned}
$$

where we have explicitly written the dependence of the co-chains $\omega_{\left(I_{0} \ldots I_{q}\right)}^{n-q}\left(x, Z_{0}\right)$ upon $x^{i}$ and $Z_{0}$, in order to recall that the remaining integrals are over the coordinates (restricted to the intersections) $Z_{0}$. Thus, the present formula takes into account all jumps of the path integral given by the naive definition. The integration over $\mathcal{C}_{I_{0} \ldots I_{q}}$ is the integration over the intersection $\mathcal{U}_{I_{0} \ldots I_{q}}$ obtained by covering the complete target space $\mathcal{M}$ with the partition of unity.

The final form of the integral of $\omega_{\text {global }}$ depends upon the choice of the partition of unity. Therefore one can choose it in the most convenient way (for instance it can be chosen such that the global form rapidly vanishes at infinity 4 or avoiding possible poles). Furthermore, the partition of unity depends upon the complex conjugate of the coordinates $Z_{I}^{i}$ and consequently the global form turns out to be non-holomorphic.

\section{$5 \quad$ Examples}

Here, we discuss some example. For some of them, we provide the construction of the measure using both the algebraic construction and the collating formula.

\subsection{Cone in $\mathbb{C}^{3}$}

Let us consider the surface in $\mathbb{C}^{3}$

$$
Z_{0}^{n}+Z_{1}^{n}+Z_{2}^{n}=0
$$

which describes a non-compact complex hypersurface. The algebraic equation is homogenous and therefore it describes a cone over a compact hypersurface in $\mathbb{P}^{2}$. According to

\footnotetext{
${ }^{4}$ For the pure spinor integration measure, this property replaces the regularization at boundary of the pure spinor space discussed in [2] while for the regularization at the poles $\lambda \cdot \bar{\lambda}=0$ the regulator 8 can be used.
} 
the previous discussion, the integration measure is given by

$$
\Omega^{(2 \mid 0)}=\frac{1}{2} \frac{\epsilon^{i j k} \bar{Z}_{k} d Z_{i} \wedge d Z_{j}}{\sum_{i=0}^{2} \bar{Z}_{i} Z_{i}^{(n-1)}}
$$

This surface can be covered by 3 patches $\mathcal{U}_{i}$, such that $Z_{i} \neq 0$. An easy computation shows that in $\mathcal{U}_{0}$, using the coordinates

$$
\phi_{(0)}^{0}=\lambda=Z_{0}, \quad \phi_{(0)}^{1}=\gamma=\frac{Z_{1}}{Z_{0}}, \quad \phi_{(0)}^{2}=u=\frac{Z_{2}}{Z_{0}}=i \sqrt{1+\gamma^{n}}
$$

and choosing a suitable $\bar{Z}_{i}$ we get

$$
\Omega^{(2 \mid 0)}=\frac{\partial \lambda}{\lambda^{(n-2)}} \frac{\partial \gamma}{u^{(n-1)}}
$$

which is holomorphic and nowhere vanishing; moreover, the factor $\frac{\partial \gamma}{u^{(n-1)}}$ is nonsingular if $n=3$, and indeed this is the holomorphic form for the CY $\mathcal{P}^{3} /\left\{\gamma^{2}+u^{2}+1=0\right\}$.

For the same space we construct the measure using the collating formula as discussed above. For that purpose, we organize the coordinates as follows: on the patch $\mathcal{U}_{0}$ we use the coordinates given in (5.3). On the the patch $\mathcal{U}_{1}$ and $\mathcal{U}_{2}$ we set

$$
\phi_{(1)}^{0}=\lambda^{\prime}=Z_{1}, \quad \phi_{(1)}^{1}=\gamma^{\prime}=\frac{Z_{0}}{Z_{1}}, \quad \phi_{(1)}^{2}=u^{\prime}=\frac{Z_{2}}{Z_{1}}
$$

and

$$
\phi_{(2)}^{0}=\lambda^{\prime \prime}=Z_{2}, \quad \phi_{(2)}^{1}=\gamma^{\prime \prime}=\frac{Z_{0}}{Z_{2}}, \quad \phi_{(2)}^{2}=u^{\prime \prime}=\frac{Z_{1}}{Z_{2}}
$$

the transition functions between the patches $\mathcal{U}_{1}$ and $\mathcal{U}_{0}$ are

$$
\lambda^{\prime}=\lambda \gamma, \quad \gamma^{\prime}=1 / \gamma, \quad u^{\prime}=u \gamma
$$

and those between $\mathcal{U}_{2}$ and $\mathcal{U}_{0}$

$$
\lambda^{\prime \prime}=\lambda u, \quad \gamma^{\prime \prime}=\gamma / u . \quad u^{\prime \prime}=1 / u
$$

Since the coordinate $\phi_{(I)}^{2}$ is always fixed by the constraint (5.1) we can take as independent variables the first two coordinates $\phi_{(I)}^{i}$ with $i=0,1$, and therefore we introduce only for them the corresponding ghost fields $\bar{C}^{i}$ and the Lagrange multipliers $B^{i}$.

In the present case we have to compute the following terms: the 2 -forms $\omega_{I}^{(2)}$, where $I=0,1,2$, the 1 -forms $\omega_{[I J]}^{(1)}$ on the intersections $\mathcal{U}_{01}, \mathcal{U}_{12}, \mathcal{U}_{02}$, and the 0 -form $\omega_{012}$ in the 
triple intersection $\mathcal{U}_{012}$. We have

$$
\begin{aligned}
\omega_{I}^{(2)} & =e^{B_{i}\left(x^{i}-\phi_{(I)}^{i}\right)+\bar{C}_{i} d \phi_{(I)}^{i}} \\
\omega_{I J}^{(1)} & =\bar{C}_{i}\left(\phi_{(J)}^{i}-\phi_{(I)}^{i}\right) \int_{0}^{1} \int_{0}^{1} d t_{I} d t_{J} e^{d\left[t_{I} \bar{C}_{i}\left(x^{i}-\phi_{(I)}^{i}\right)+t_{J} \bar{C}_{i}\left(x^{i}-\phi_{(J)}^{i}\right)\right]} \delta\left(t_{I}+t_{J}-1\right) \\
\omega_{I J K}^{(0)} & =\bar{C}_{i} \bar{C}_{j}\left(\phi_{(I)}^{i} \phi_{(J)}^{j}+\phi_{(J)}^{i} \phi_{(K)}^{j}+\phi_{(K)}^{i} \phi_{(I)}^{j}\right) \\
& \times \int_{0}^{1} \int_{0}^{1} \int_{0}^{1} d t_{I} d t_{J} d t_{K} e^{d\left[\sum_{m=0}^{2} t_{I m} \bar{C}_{i}\left(x^{i}-\phi_{(I m)}^{i}\right)\right]} \delta\left(\sum_{m=0}^{2} t_{I_{m}}-1\right)
\end{aligned}
$$

which satisfy the descent equations:

$$
d \omega_{I}^{(2)}=0,\left(\delta \omega^{(2)}\right)_{I J}=d \omega_{I J}^{(1)},\left(\delta \omega^{(1)}\right)_{I J K}=d \omega_{I J K}^{(0)},\left(\delta \omega^{(0)}\right)_{I J K}=0,
$$

From these equations, acting with the homotopy operator, we can reconstruct the global form. Using the partition of unity

$$
\rho_{0}=\frac{1}{\Delta}, \quad \rho_{1}=\frac{|\gamma|^{2}}{\Delta}, \quad \rho_{2}=\frac{|u|^{2}}{\Delta}, \quad \Delta=1+|\gamma|^{2}+|u|^{2}
$$

upon integration over the ghost fields $\bar{C}^{i}$, over the auxiliary fields $B^{i}$ and over the $t^{\prime}$ s, we get the following global 2 -form

$$
\begin{aligned}
\Omega^{(2 \mid 0)}= & \frac{1}{2 \Delta^{2}}\left[2 \Delta\left(1-\frac{|\gamma|^{2}}{\gamma}-\frac{|u|^{2}}{u^{3}}\right)+\bar{\gamma}(1+\gamma)\left(\gamma-\frac{1}{\gamma}\right)-\frac{\bar{u} \gamma^{2}}{u^{3}}\left(u^{2}-1\right)+\right. \\
& \left.-\left(|\gamma|^{2} \frac{\bar{u} \gamma^{2}}{u^{2}}-|u|^{2} \bar{\gamma}\right)(\gamma+u)\left(\frac{1}{\gamma}-\frac{\gamma}{u}\right)\right] \partial \lambda \partial \gamma,
\end{aligned}
$$

The form (5.12) is globally defined and it is not singular. It is not holomorphic due to the non-holomorphicity of the partition of unity. In addition, the ratio between the present formula and the holomorphic one (in the case of $n=3$ ) is a globally defined function on the hypersurface.

Notice that, in the present example, we have set to zero the action $S[x]$ and the vertex operators $\mathcal{O}_{A}(x, p)$ introduced in the previous section. This simplified the construction. Without setting them to zero, the result is definitely more interesting and complicate.

\subsection{Kähler form Collating Formula}

The next example is the construction, via the collating formula, of a global 2 form for the projective space $\mathbb{P}^{2}$. We first will do it in a simplified way by starting from a real form on the different patches and, in the second place, we will employ the complete construction 
given in the previous section by starting from a holomorphic 2 form on the different patches. The two resulting expressions will differ for a globally defined function.

Let us consider $\mathbb{C} \mathbb{P}^{n}$. It can be covered by $n+1$ open sets $\mathcal{U}_{I}$, where homogeneous coordinates $\gamma_{J}^{(I)}=\frac{z_{J}}{z_{I}}$ can be defined (clearly there are only $n$ independent coordinates, since by definition $\gamma_{I}^{(I)}=1$ ). In the intersection between two patches $\mathcal{U}_{I J} \equiv \mathcal{U}_{I} \cap \mathcal{U}_{J}$ the following relation holds:

$$
\gamma_{K}^{(J)}=\frac{\gamma_{K}^{(I)}}{\gamma_{J}^{(I)}}
$$

In any patch $\mathcal{U}_{I}$ a real $2-$ form can be defined:

$$
\omega_{I}=\sum_{J=1}^{n+1}\left|\partial \gamma_{J}^{(I)}\right|^{2}=\frac{1}{2} d \sum_{J=1}^{n+1}\left[\gamma_{J}^{(I)} \bar{\partial} \bar{\gamma}_{J}^{(I)}-\bar{\gamma}_{J}^{(I)} \partial \gamma_{J}^{(I)}\right]=\frac{1}{2} d \sum_{J=1}^{n+1}\left|\gamma_{J}^{(I)}\right|^{2} \mu_{J}^{(I)}
$$

where

$$
\mu_{J}^{(I)}=\frac{\bar{\partial} \bar{\gamma}_{J}^{(I)}}{\bar{\gamma}_{J}^{(I)}}-\frac{\partial \gamma_{J}^{(I)}}{\gamma_{J}^{(I)}}
$$

The 1 -forms $\mu_{J}^{(I)}$ have some interesting properties: they are $d$-closed and, changing from patch $\mathcal{U}_{I}$ to patch $\mathcal{U}_{J}$, they transform according to

$$
\mu_{K}^{(I)}=\mu_{K}^{(J)}-\mu_{I}^{(J)}
$$

Using the descent equation

$$
(\delta \omega)_{I J} \equiv \omega_{I}-\omega_{J}=d \omega_{I J}
$$

it is easy to find that

$$
\omega_{I J}=\frac{1}{2} \sum_{K=1}^{n+1}\left[\left|\gamma_{K}^{(I)}\right|^{2}\left(\frac{\left|\gamma_{J}^{(I)}\right|^{2}-1}{\left|\gamma_{J}^{(I)}\right|^{2}}\right) \mu_{K}^{(I)}+\frac{\left|\gamma_{K}^{(I)}\right|^{2}}{\left|\gamma_{J}^{(I)}\right|^{2}} \mu_{J}^{(I)}\right]
$$

while, in the triple intersection $\mathcal{U}_{I J K}$, all the $\omega_{I J K}$ vanish. It is useful to take, as partition of unity, the expression

$$
\rho^{J}=\frac{\left|\gamma_{J}^{(I)}\right|^{2}}{\sum_{K=1}^{n+1}\left|\gamma_{K}^{(I)}\right|^{2}}
$$

Plugging everything in (4.11), we find that the globally defined 2-form is

$$
\Lambda=\frac{n+1}{2} d \frac{\sum_{J}\left|\gamma_{J}^{(I)}\right|^{2} \mu_{J}^{(I)}}{\sum_{K}\left|\gamma_{K}^{(I)}\right|^{2}}
$$

But since, as it is evident from eq. (5.15)

$$
\left|\gamma_{J}^{(I)}\right|^{2} \mu_{J}^{(I)}=(\bar{\partial}-\partial)\left|\gamma_{J}^{(I)}\right|^{2}
$$


while $d=(\bar{\partial}+\partial)$, it turns out that

$$
\Lambda=(n+1) \partial \bar{\partial} \log \sum_{K=1}^{n+1}\left|\gamma_{K}\right|^{2}=(n+1) K
$$

As we anticipated, we now derive a global 2-form starting from the 2-forms on the three patches

$$
\omega_{0}=\partial u \partial v, \quad \omega_{1}=-\frac{1}{u^{3}} \partial u \partial v, \quad \omega_{2}=-\frac{1}{v^{3}} \partial u \partial v
$$

Using the formula (4.12) we can compute the 2-cochain living on the simple intersections

$$
\begin{aligned}
\omega_{01} & =\frac{1}{2}\left(1-\frac{1}{u^{2}}\right)[(1+u) \partial v-v \partial u] \\
\omega_{12} & =\frac{1}{2}\left[\left(\frac{1}{u^{2}}-\frac{1}{u v^{2}}\right) \partial v-\left(\frac{1}{v^{2}}-\frac{1}{v u^{2}}\right) \partial u\right] \\
\omega_{20} & =\frac{1}{2}\left(1-\frac{1}{v^{2}}\right)[(1+v) \partial u-u \partial v]
\end{aligned}
$$

and finally the 0-form living on the triple intersection

$$
\omega_{012}=\left(u+v+\frac{1}{u v}-\frac{u}{v}-\frac{v}{u}-1\right)
$$

After some simple algebraic manipulation, (4.11) can be rewritten as

$$
\omega_{0}^{g l}=\omega_{o}+d \omega_{20} \rho_{2}-d \omega_{01} \rho_{1}-\omega_{20} d \rho_{2}+\omega_{01} d \rho_{1}+2 \omega_{012} d \rho_{1} d \rho_{2}-d \omega_{012}\left(d \rho_{1} \rho_{2}-d \rho_{2} \rho_{1}\right)
$$

therefore, by inserting the different pieces we arrive at the cumbersome expression

$$
\begin{aligned}
\omega_{0}^{g l} & =\partial u \partial v+\left(-\frac{|v|^{2}}{\Delta}\left(1+\frac{1}{v^{3}}\right)-\frac{|u|^{2}}{\Delta}\left(1+\frac{1}{u^{3}}\right)\right) \partial u \partial v+ \\
& -\frac{1}{2 \Delta^{2}}\left[\left(\frac{1}{u^{2}}-\frac{1}{u v^{2}}\right) \partial v+\left(\frac{1}{v^{2}}-\frac{1}{v u^{2}}\right) \partial u\right]\left[|v|^{2}(u \bar{\partial} \bar{u}+\bar{u} \partial u)-|u|^{2}(v \bar{\partial} \bar{v}+\bar{v} \partial v)\right]+ \\
& +\frac{1}{2 \Delta^{3}}\left(1-\frac{1}{u^{2}}\right)[(1+u) \partial v-v \partial u]\left[\left(1+|u|^{2}\right)(v \bar{\partial} \bar{v}+\bar{v} \partial v)-|v|^{2}(u \bar{\partial} \bar{u}+\bar{u} \partial u)\right]+ \\
& -\frac{1}{2 \Delta^{3}}\left(1-\frac{1}{v^{2}}\right)[(1+v) \partial u-u \partial v]\left[\left(1+|v|^{2}\right)(u \bar{\partial} \bar{u}+\bar{u} \partial u)-|u|^{2}(v \bar{\partial} \bar{v}+\bar{v} \partial v)\right]+ \\
& +\frac{2}{\Delta^{3}}\left(u+v+\frac{1}{u v}-\frac{u}{v}-\frac{v}{u}-1\right)(u \bar{\partial} \bar{u}+\bar{u} \partial u)(v \bar{\partial} \bar{v}+\bar{v} \partial v)
\end{aligned}
$$

In order to compare the two results, we have to evaluate $\Lambda \wedge \Lambda$ (in the case $n=2$ ), and $\omega_{0}^{g l} \wedge \bar{\omega}_{0}^{g l}$; the results are proportional up to a globally defined function. 


\subsection{Pure Spinors}

Let us recall some basic fact about the pure spinors. We consider a spinor $\lambda^{\alpha}$ in $10 \mathrm{~d}$ satisfying the algebraic equation $W^{m}(\lambda)=\lambda^{\alpha} \gamma_{\alpha \beta}^{m} \lambda^{\beta}=0$ where $m=0, \ldots, 9$. This is not a complete intersection since the constraints $W^{m}(\lambda)=0$ are not independent from each others. The coordinates of the PS space are $\lambda^{+}, u_{a}$ and $u^{a b}\left(a=1 \ldots 5\right.$, and $\left.u^{a b}=-u^{b a}\right)$, constrained by the relation $u_{a}=\frac{1}{8} \epsilon_{a b c d e} u^{b c} u^{d e}$ which solve the pure spinor condition. Correspondingly there are 16 patches:

$$
\underbrace{\mathcal{U}_{+}}_{1}, \underbrace{\mathcal{U}_{a}}_{5}, \underbrace{\mathcal{U}_{a b}}_{10}
$$

The transition functions allowing us to move from a patch to another one can be found in [4]; we only quote those needed for our purpose, that is those that tell us how to move from the patch $\mathcal{U}_{a}$ or $\mathcal{U}_{a b}$ to $\mathcal{U}_{+}$:

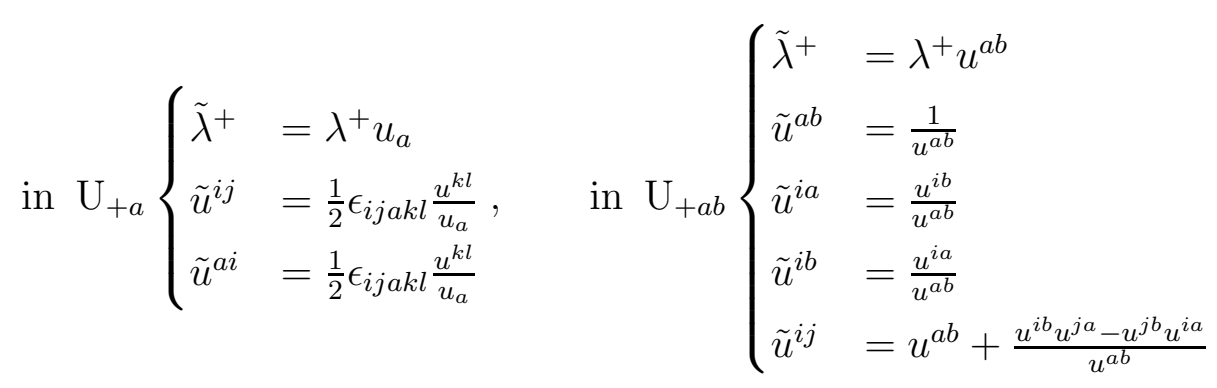

We have to find 11 independent globally defined 1 -forms, so that we can use the collating formula to globalize $d \lambda^{+}$and $d u^{a b}$. The result is:

$$
\Lambda_{g l o b}=d\left(\frac{\lambda^{+}\left(1+\sum_{a=1}^{5} u_{a}^{2} \bar{u}^{a}+\frac{1}{2} \sum_{a, b=1}^{5} u^{a b^{2}} \bar{u}_{a b}\right)}{1+\sum_{a=1}^{5} u_{a} \bar{u}^{a}+\frac{1}{2} \sum_{a, b=1}^{5} u^{a b} \bar{u}_{a b}}\right)
$$

and

$\Sigma_{g l o b}^{a b}=d\left(\frac{u^{a b}+u^{a b} u^{i j} \bar{u}_{i j}+\frac{1}{2}\left(\bar{u}^{a} u^{a i}+\bar{u}^{b} u^{b i}\right) u^{j k} \epsilon_{a b i j k}+\bar{u}_{a b}+u^{a i} \bar{u}_{b i}-u^{b i} \bar{u}_{a i}+2 u^{a i} \bar{u}_{i j} u^{j b}}{1+\sum_{a=1}^{5} u_{a} \bar{u}^{a}+\frac{1}{2} \sum_{a, b=1}^{5} u^{a b} \bar{u}_{a b}}\right)$

The global form is obtained by computing the wedge product of the 11 forms and by multiplying it with its conjugate

$$
\Omega^{(11)}=\Lambda_{g l o b} \bigwedge_{a<b} \Sigma_{g l o b}^{a b} \wedge \overline{\Lambda_{g l o b} \bigwedge_{a<b} \Sigma_{g l o b}^{a b}}
$$

which should be normalized by $\int \Omega^{(11)}$ to get the correct value for physical amplitudes. 


\subsection{Grassmannian $G(2,4)$}

Grassmannians $G(k, n)$ are the set of $k$-planes in $\mathbb{C}^{n}$. They are an algebraic variety in $\mathbb{C P}\left(\begin{array}{l}n \\ k\end{array}\right)-1$, identified by the Plücker relations. For any $G(k, n)$ we can define the $\left(\begin{array}{l}n \\ k\end{array}\right)$ so-called Plücker coordinates $\lambda_{\left[i_{1} \ldots i_{k}\right]}$ which give explicitly the embedding. For instance, $G(2,4)$ is the algebraic variety in $\mathbb{C P}^{5}$ given by the relation $\epsilon^{a b c d} \lambda_{a b} \lambda_{c d}(a, b: 1 \ldots 4$ and $\left.\lambda_{b a}=-\lambda_{a b}\right)$. It can be covered by 6 patches: $\mathcal{U}_{i j}$ such that $\lambda_{i j} \neq 0$.

In $\mathcal{U}_{0}$ (where $\lambda_{12} \neq 0$ ) we can rename the coordinates as:

$$
\begin{array}{lll}
\lambda_{12}=\gamma, & \lambda_{13}=\gamma x, & \lambda_{14}=\gamma y \\
\lambda_{23}=\gamma z, & \lambda_{24}=\gamma w, & \lambda_{34}=\gamma t
\end{array}
$$

where $t=x w-y z$ is a dependent coordinate, due to the Plücker relation. After repeating the procedure for all the other patches and defining the following partition of unity

$$
\begin{aligned}
& \Delta=1+|x|^{2}+|y|^{2}+|z|^{2}+|w|^{2}+|t|^{2} \\
& \rho_{0}=\frac{1}{\Delta}, \rho_{x}=\frac{|x|^{2}}{\Delta}, \rho_{y}=\frac{|y|^{2}}{\Delta} \\
& \rho_{z}=\frac{|z|^{2}}{\Delta}, \rho_{w}=\frac{|w|^{2}}{\Delta}, \rho_{t}=\frac{|t|^{2}}{\Delta}
\end{aligned}
$$

the collating formula can be used to globalize the following 4 independent 1 -forms

$$
\begin{aligned}
& \omega_{1}=d x \rightarrow \Omega_{1}^{G l o b}=d\left[\frac{x(1+\bar{y}+\bar{z}+\bar{t})+\bar{x}+t \bar{w}}{\Delta}\right] \\
& \omega_{2}=d y \rightarrow \Omega_{2}^{G l o b}=d\left[\frac{y(1+\bar{w}-\bar{x}+\bar{t})+\bar{y}+t \bar{z}}{\Delta}\right] \\
& \omega_{3}=d z \rightarrow \Omega_{3}^{G l o b}=d\left[\frac{z(1+\bar{x}-\bar{w}+\bar{t})+\bar{z}+t \bar{y}}{\Delta}\right] \\
& \omega_{4}=d w \rightarrow \Omega_{4}^{G l o b}=d\left[\frac{w(1+\bar{y}+\bar{z}+\bar{t})+\bar{w}+t \bar{x}}{\Delta}\right]
\end{aligned}
$$

Alternatively we could have started from the 4 -form

$$
\sigma=4 d x d y d z d w \equiv 4 \Lambda
$$

which would have led to the following globally defined 4-form

$$
\Sigma=\frac{1}{\Delta^{2}}\left[\Delta\left(\frac{\partial f^{x}}{\partial x}+\frac{\partial f^{y}}{\partial y}+\frac{\partial f^{z}}{\partial z}+\frac{\partial f^{w}}{\partial w}\right)-\left(\frac{\partial \Delta}{\partial x} f^{x}+\frac{\partial \Delta}{\partial y} f^{y}+\frac{\partial \Delta}{\partial z} f^{z}+\frac{\partial \Delta}{\partial w} f^{w}\right)\right] \Lambda
$$


where the $f$ functions are defined to be

$$
\begin{aligned}
& f^{x}=x\left(1+\frac{|y|^{2}}{y^{4}}+\frac{|z|^{2}}{z^{4}}+\frac{|w|^{2}}{w^{4}}\right)-\frac{|x|^{2}}{3 x^{3}}+\frac{|t|^{2}}{3 t^{3} w} \\
& f^{y}=y\left(1+\frac{|x|^{2}}{x^{4}}+\frac{|z|^{2}}{z^{4}}+\frac{|w|^{2}}{w^{4}}\right)-\frac{|y|^{2}}{3 y^{3}}+\frac{|t|^{2}}{3 t^{3} z} \\
& f^{z}=z\left(1+\frac{|x|^{2}}{x^{4}}+\frac{|y|^{2}}{y^{4}}+\frac{|w|^{2}}{w^{4}}\right)-\frac{|z|^{2}}{3 z^{3}}+\frac{|t|^{2}}{3 t^{3} y} \\
& f^{w}=w\left(1+\frac{|x|^{2}}{x^{4}}+\frac{|y|^{2}}{y^{4}}+\frac{|z|^{2}}{z^{4}}\right)-\frac{|w|^{2}}{3 w^{3}}+\frac{|t|^{2}}{3 t^{3} x}
\end{aligned}
$$

\section{Conclusions}

We have used a method developed some years ago in the context of topological gravity and topological string theory to construct globally defined forms. It uses the collating formula and we have adapted the method to pure spinor string theory and related theories based on algebraic manifolds. We generalized the Griffiths' residue method and we derived a solution of the descent equations. Some examples are presented and the present paper is in preparation of more physical applications, such as amplitude computations in string theory.

\section{Acknowledgments}

We are grateful to D. Matessi for several discussions on algebraic geometry.

\section{Bibliography}

[1] N. Berkovits, "Multiloop amplitudes and vanishing theorems using the pure spinor formalism for the superstring," JHEP 0409 (2004) 047. hep-th/0406055.

[2] N. Berkovits, N. Nekrasov, "Multiloop superstring amplitudes from non-minimal pure spinor formalism," JHEP 0612 (2006) 029. hep-th/0609012.

[3] O. A. Bedoya, N. Berkovits, "GGI Lectures on the Pure Spinor Formalism of the Superstring," arXiv:0910.2254 [hep-th]].

[4] N. A. Nekrasov, "Lectures on curved beta-gamma systems, pure spinors, and anomalies," hep-th/0511008. 
[5] H. Gomez, "One-loop Superstring Amplitude From Integrals on Pure Spinors Space," JHEP 0912 (2009) 034. arXiv:0910.3405 [hep-th]].

[6] H. Gomez, C. R. Mafra, "The Overall Coefficient of the Two-loop Superstring Amplitude Using Pure Spinors," JHEP 1005 (2010) 017. arXiv:1003.0678 [hep-th]].

[7] J. Hoogeveen, K. Skenderis, "Decoupling of unphysical states in the minimal pure spinor formalism I," JHEP 1001 (2010) 041. [arXiv:0906.3368 [hep-th]].

[8] P. A. Grassi, P. Vanhove, "Higher-loop amplitudes in the non-minimal pure spinor formalism," JHEP 0905 (2009) 089. [arXiv:0903.3903 [hep-th]].

[9] C. I. Lazaroiu, "Holomorphic matrix models," JHEP 0305 (2003) 044. hep-th/0303008].

[10] N. Berkovits, "Pure spinor formalism as an $\mathrm{N}=2$ topological string," JHEP 0510 (2005) 089. hep-th/0509120.

[11] R. Bott and L. Tu, "Differential Form in Algebraic Topology," GTM Springer, 1982.

[12] Y. Aisaka, N. Berkovits, "Pure Spinor Vertex Operators in Siegel Gauge and Loop Amplitude Regularization," JHEP 0907 (2009) 062. arXiv:0903.3443 [hep-th]].

[13] I. Y. Park, "Pure spinor computation towards open string three-loop," JHEP 1009 (2010) 008. arXiv:1003.5711 [hep-th]].

[14] J. Bjornsson, M. B. Green, "5 loops in 24/5 dimensions," JHEP 1008 (2010) 132. arXiv:1004.2692 [hep-th]].

[15] C. M. Becchi, C. Imbimbo, "Gribov horizon, contact terms and Cech-De Rham cohomology in 2-D topological gravity," Nucl. Phys. B462 (1996) 571-599. hep-th/9510003.

[16] P. Griffiths and J. Harris, "Principles of Algebraic Geometry," Wiley-Interscience, 1994.

[17] N. Berkovits, J. Hoogeveen, K. Skenderis, "Decoupling of unphysical states in the minimal pure spinor formalism II," JHEP 0909 (2009) 035. arXiv:0906.3371 [hepth]].

[18] C. Becchi, "Introduction to BRS symmetry," arXiv:hep-th/9607181. 
[19] C. M. Becchi, S. Giusto, C. Imbimbo, "Gauge dependence in topological gauge theories," Phys. Lett. B393 (1997) 342-348. hep-th/9611113. 